\title{
Hemostatic analysis of dogs naturally envenomed by the African puffadder (Bitis arietans) and snouted cobra (Naja annulifera)
}

\author{
Susanna S. Nagel, BVSc; Johan P. Schoeman, BVSc, MMedVet, PhD, DSAM, DECVIM; \\ PeterN.Thompson,BVSc,MMedVet,PhD;BoWiinberg,DVM,PhDandAmelia Goddard,BVSc, MMedVet
}

\begin{abstract}
Objective - To investigate hemostatic changes in dogs envenomed by cytotoxic (African puffadder) and neurotoxic snakes (snouted cobra) using thromboelastography (TEG) and plasma-based coagulation assays.

Design - Prospective observational clinical study.

Setting - University teaching hospital.

Animals - Eighteen client-owned dogs; 9 envenomed by African puffadder (Bitis arietans) and 9 by snouted cobra (Naja annulifera). Ten healthy dogs served as controls.

Interventions - None.

Measurements and Main Results - Blood was collected at presentation and 24 hours post envenomation. Platelet count, TEG, prothrombin time, activated partial thromboplastin time (aPTT), antithrombin activity, and fibrinogen (Fib) and C-reactive protein (CRP) concentrations were measured. Outcomes were analyzed using linear mixed models at 5\% significance. At presentation, $R$ time was significantly prolonged in the puffadder group compared to the cobra $(P=0.01)$ and control groups $(P=0.05)$. Platelet count was significantly lower in the puffadder compared to the cobra $(P=0.04)$ and control groups $(P=0.001)$, respectively. Antithrombin activity was significantly decreased in the puffadder $(P=0.002)$ and cobra groups $(P=0.004)$ compared to the control group. Both prothrombin time and activated partial thromboplastin time were significantly prolonged in the cobra group compared to the control group ( $P=0.03$ for both). The TEG variables, maximum amplitude (MA) and $G$, were significantly increased 24 hours post envenomation in the puffadder group compared to their values at presentation ( $P=0.05$ for both). Fib and CRP concentrations were significantly increased 24 hours post envenomation in both snake-envenomed groups.

Conclusions - Prolonged clot initiation was a common feature in puffadder-envenomed dogs at presentation and this was likely venom induced. Snouted cobra-envenomed dogs were normo- to hypercoagulable at presentation. Dogs from both puffadder and cobra groups progressed to a more hypercoagulable by 24 hours post envenomation, most likely due to marked inflammation as indicated by the increased Fib and CRP concentrations. TEG proved a sensitive tool for detecting abnormal hemostasis in snake-envenomed dogs.
\end{abstract}

Keywords: venom, snake, coagulopathy, thrombocytopenia, thromboelastography

From the Departments of Companion Animal Clinical Studies (Nagel, Schoeman, Goddard), Department of Production Animal Studies (Thompson), Faculty of Veterinary Science, University of Pretoria, Onderstepoort, South Africa; and Department of Small Animal Clinical Sciences, Faculty of Life Sciences, University of Copenhagen (Wiinberg).

Dr. Wiinberg's current address: Translational Haemophilia Pharmacology, Biopharmaceuticals Research Unit, Novo Nordisk A/S, Denmark.

The authors declare no conflict of interest.

This study was presented in part at the European College of Veterinary Internal Medicine (ECVIM) Congress in Seville, Spain in September 2011.

Address correspondence and reprint requests to

Professor Amelia Goddard, Department of Companion Animal ClinicalStudies, Faculty of Veterinary Science, University of Pretoria, Private Bag X04, Onderstepoort 0110, South Africa. Email: amelia.goddard@up.ac.za

Submitted February 22, 2013; Accepted August 19, 2014.
Abbreviations
aPTT activated partial thromboplastin time
AT antithrombin
CRP C-reactive protein
Fib fibrinogen
HCT hematocrit
Plt platelet count
PT prothrombin time
TEG thromboelastography 


\section{Introduction}

Snake envenoming in people and animals is a common occurrence in many countries across the world, especially in rural areas, and is classified as a true medical emergency. ${ }^{1-4}$ Snake venom contains hundreds of enzymes, proteins, and peptides that assist the snake in paralyzing, killing, and digesting its prey or to defend itself against predators. ${ }^{3,5-7}$ These venom components can be classified into procoagulants, anticoagulants, fibrinolytic toxins, vessel wall interactive toxins, toxins that affect platelet activity and plasma protein activators. ${ }^{8}$ These not only destroy tissues, but can also evoke a significant inflammatory response through cytokine release in the victim. ${ }^{9,10}$ In viperine (adders) and crotaline (rattlesnakes) species, inflammation is a major characteristic of envenoming and inflammation and coagulation are known to inadvertently activate each other. ${ }^{11-13}$

Plasma-based assays are routinely used in human medicine to screen for coagulopathies in envenomed patients. ${ }^{8}$ The usefulness of these assays is limited because they do not take into account the role of platelets, the endothelium, or the contribution of tissue-factor bearing cells toward hemostasis. Thromboelastography (TEG) is a possible alternative to characterize the hemostatic changes following envenoming in dogs by incorporating the cellular elements, which are important in the inflammatory response. It is believed that TEG offers a better reflection of hemostasis in vivo and it has been validated and used in dogs. ${ }^{14-19}$ One study reported on the usefulness of TEG in the clinical management of children with snake envenomation in southern Africa. ${ }^{20}$ Thirtyfive of the 51 patients had abnormal thromboelastograms and 17 of those 35 patients developed severe hemorrhagic diathesis. The study concluded that in snake envenoming, $50 \%$ of the patients with abnormal thromboelastograms will develop a severe clinical diathesis, while if the thromboelastogram is normal, the clinical course is likely to be benign (predictive value of $94 \%$ ). Patients with an abnormal thromboelastogram should therefore be monitored for clinical signs of a coagulopathy. ${ }^{20}$

Three snake families are considered to have clinically relevant effects on hemostasis: Colubridae (eg, boomslang and vine snake), Elapidae (eg, cobras and mambas), and Viperidae (eg, rattlesnakes and vipers, such as the puffadder). ${ }^{6,8}$ The African puffadder (Bitis arietans) and snouted cobra (Naja annulifera) are typically found in sub-Saharan Africa and envenomations by these snakes are commonly seen in both people and animals. ${ }^{21,22}$ Even though the hematological and biochemical findings in dogs envenomed by these 2 snakes have been documented, ${ }^{23}$ possible hemostatic dysfunction in dogs naturally envenomed by them has, to our knowledge, not been investigated. An understanding of the hemostatic changes may be of value in the diagnosis, management, and prognosis of envenomed patients. The purpose of this study was therefore to investigate the hemostatic changes in dogs envenomed by 2 snake species (B. arietans and $N$. annulifera) using TEG and traditional plasma-based coagulation assays.

\section{Materials and Methods}

\section{Animals}

This was a prospective, descriptive, longitudinal observational study of clinical cases of 18 client-owned dogs with confirmed natural snake envenomation. Eligible dogs were of any breed and either sex, provided they were $\geq 6$ months of age, weighed more than $5 \mathrm{~kg}$, and were free of any concurrent diseases that may affect hemostasis as determined by thorough physical examination and comprehensive blood assays. The presence of blood-borne parasites (ie, Ehrlichia spp. or Babesia spp.) was screened for by careful examination of a thin peripheral blood smear and CBC. Dogs that had received medication known to interfere with hemostasis (eg, corticosteroids, nonsteroidal anti-inflammatory drugs, or anticoagulants) within the preceding 3 weeks were excluded from the study. To ensure correct snake identification, the envenomation had to have been witnessed by the owner and the snake identified by means of either an accurate description, using pictures compiled and kept at the clinic, or on presentation of the dead snake. The time from envenomation to presentation was recorded. All envenomed dogs were treated according to the specifications of a previous publication as deemed necessary by the attending clinician, and included IV fluids, blood products, polyvalent antivenom, and other supportive treatments as indicated by the patient's clinical signs. ${ }^{4}$ Specific treatments that may have interfered with hemostasis (eg, blood products or polyvalent antivenom) were recorded. The polyvalent antivenom ${ }^{a}$ consists of refined equine serum immunoglobulins, prepared from the serum of horses that have been hyperimmunized with specific snake venoms. The controls were selected to be in the same age and weight range as the clinical cases, and included 10 healthy, clientowned dogs admitted for routine ovariohysterectomy, castration, or blood donation. The controls were deemed healthy based on history, a full clinical examination that included a peripheral blood smear to screen for bloodborne parasites, as well as a CBC. Client consent was obtained for all dogs included in this study and the study protocol was approved by the Animal Ethics Committee of the University of Pretoria (Protocol no. V058-10). 


\section{Sample collection and analysis}

Envenomed dogs were presented at different times after the incident occurred. In an attempt to standardize the time of blood collection, dogs were only recruited if they presented within 6 hours post envenomation. Blood was collected at presentation prior to any treatment, as well as 24 hours post envenomation. A serum sample (3-mL Vacutainer tube), ${ }^{\text {b }}$ sodium citrate sample (3-mL Vacutainer tube), ${ }^{\mathrm{b}}$ and an EDTA sample (3-mL Vacutainer tube $)^{b}$ were collected from the jugular vein of each dog with a 21-Ga needle by careful venipuncture with minimum stasis. Sufficient blood was collected in the citrate tube to ensure a 1:9 ratio of $3.2 \%$ trisodium citrate and blood. The blood samples were collected in the order described above. The EDTA sample was used to determine platelet count (Plt), hematocrit (HCT), and white blood cell count (WBC). The TEG analysis was performed on the sodium citrate sample 30 minutes after collection. The remaining citrate sample was then centrifuged at 2,100 $\times g$ for 8 minutes, after which the plasma was harvested and stored at $-80^{\circ} \mathrm{C} .^{\mathrm{c}}$ The coagulation profile analyses were performed as a batch within 4 months of collection and included prothrombin time (PT), activated partial thromboplastin time (aPTT), antithrombin (AT) activity, and fibrinogen (Fib) concentration. Studies in people and dogs have reported that coagulation proteins in frozen plasma remain stable between 6 and 24 months at $-70^{\circ} \mathrm{C} .{ }^{24,25} \mathrm{~A} \mathrm{CBC}$ was performed on the ADVIA 2120. ${ }^{\mathrm{d} T}$, aPTT, and Fib concentration assays were performed on the ST art 4 analyzer. ${ }^{e}$ AT activity was measured using a chromogenic assay on an automated spectrophotometric analyzer. ${ }^{f} \mathrm{C}$-reactive protein (CRP) concentration was measured using an automated turbidometric immunoassay calibrated with commercially available purified canine CRP to ensure species-specific measurement with the heterologous assay. ${ }^{\mathrm{f}}$ The assay has been validated for use in dogs. ${ }^{26}$ TEG was performed using the TEG 5000 Thromboelastograph Haemostasis System. ${ }^{g}$ Citrated whole blood was left to stand for 30 minutes to allow settling of the platelets' activity levels before TEG analysis. Human-recombinant tissue factor ${ }^{\mathrm{h}}$ was utilized as activator, as previously described for use in dogs. ${ }^{14}$ Thromboelastograms were obtained for 120 minutes at $37^{\circ} \mathrm{C}$. Recorded TEG variables included time to initiation of clot formation (reaction time, $R$ ), time for the tracing to achieve a predetermined clot strength $(K)$, speed of clot propagation (angle, $\alpha$ ), greatest clot strength (MA), and degree of fibrinolysis at 30 and 60 minutes after MA was reached (Ly30 and Ly60, respectively).

\section{Statistical methods}

The data were checked for normality using the ShapiroWilk test and $R, K, \mathrm{PT}$, and aPTT were log-transformed to achieve normality. Age and weight were compared between the 3 groups (puffadder, cobra, and control) using Kruskal-Wallis analysis of variance and gender proportions were compared using Fisher's exact test. TEG variables Ly30 and Ly60 were not included in the statistical analysis due to a large amount of zero values and missing values. Hematologic and hemostatic outcomes were compared between the 3 groups at admission, and between the puffadder and cobra groups over time using linear mixed models. The TEG variables $K$, angle, $\mathrm{MA}$, and $G$ were adjusted for Plt and Fib concentration at admission and 24 hours, as well as for treatment administered (polyvalent antivenom or blood products) at 24 hours. Since the $R$ time is not affected by Plt or Fib concentration, it was only adjusted for the treatment administered at 24 hours. ${ }^{14}$ The mean $\mathrm{Ht}$ remained within normal limits for all groups and therefore was not adjusted for. The remaining variables were only adjusted for treatment administered at 24 hours. Statistical analyses were performed using commercial statistical software. ${ }^{i, j}$ A $P$ value $<0.05$ was considered significant.

\section{Results}

\section{Study population}

Twenty-three envenomed dogs presented between November 2010 and April 2011. Eighteen dogs met the inclusion criteria and were enrolled in the study. Nine dogs were envenomed by African puffadder and 9 by snouted cobra. The median age (range) and weight (range) of dogs in the puffadder group were 42 months (12-144) and $10.2 \mathrm{~kg}$ (7.0-33.6), respectively, and included 4 female and 5 male dogs. The breeds consisted of smooth-haired Dachshunds (2), Jack Russell Terriers (2), Boerboels (2), and 1 of each Rhodesian Ridgeback, and crossbreed dog. The median age (range) and weight (range) for the cobra group were 42 months (18$60)$ and $17.8 \mathrm{~kg}$ (5.8-43.2), respectively, and included 6 females and 3 males. The breeds consisted of smoothhaired Dachshunds (3), and 1 each of the following, Fox Terrier, Staffordshire Bull Terrier, Bullmastiff, Boerboel, Rhodesian Ridgeback, and a crossbreed dog. The control dogs included 4 males and 6 females, with median age (range) and weight (range) of 52 months (16-96) and 17.6 $\mathrm{kg}$ (6.0-38.0), respectively. There were no significant differences in age, weight, or gender between the 3 groups.

\section{Envenomation time, treatment, and outcome}

The mean time (range) from envenomation to presentation in the puffadder and cobra groups was 2 hours (0.5-4.5) and 3 hours (0.5-4.5), respectively. Besides the indicated supportive treatment, specific treatment for the puffadder group included fresh frozen plasma 
Table 1: Descriptive statistics for hematologic and hemostatic variables in puffadder-envenomed, snouted cobra-envenomed, and control dogs at presentation and 24 hours post envenomation

\begin{tabular}{|c|c|c|c|c|c|c|c|c|c|c|}
\hline \multirow{3}{*}{$\begin{array}{l}\text { Variable } \\
\text { [SI unit (Conv unit)] } \\
\text { (Reference interval) }\end{array}$} & \multirow{2}{*}{\multicolumn{2}{|c|}{$\begin{array}{l}\text { Control group }(\mathrm{n}=10) \\
\text { Mean } \pm \mathrm{SD}(\mathrm{range}) \\
\text { Median (IQR) }\end{array}$}} & \multicolumn{4}{|c|}{$\begin{array}{l}\text { Puffadder group }(\mathrm{n}=9) \\
\text { Mean } \pm \text { SD (range) } \\
\text { Median (IQR) }\end{array}$} & \multicolumn{4}{|c|}{$\begin{array}{l}\text { Snouted cobra group }(\mathrm{n}=9) \\
\text { Mean } \pm \mathrm{SD}(\text { range) } \\
\text { Median/IQR }\end{array}$} \\
\hline & & & \multicolumn{2}{|c|}{ Presentation } & \multicolumn{2}{|l|}{24 hours } & \multicolumn{2}{|l|}{ Presentation } & \multicolumn{2}{|l|}{24 hours } \\
\hline & SI unit & Conventional unit & SI unit & Conventional unit & SI unit & Conventional unit & SI unit & Conventional unit & SI unit & Conventional unit \\
\hline $\begin{array}{l}\text { Plt }\left[\times 10^{9} / \mathrm{L}\left(\times 10^{3} / \mathrm{LL}\right)\right] \\
(200-500)\end{array}$ & $\begin{array}{l}328 \pm 76(218-455) \\
328(269-377.3)\end{array}$ & $\begin{array}{l}328 \pm 76(218-455) \\
328(269-377.3)\end{array}$ & $\begin{array}{l}136 \pm 154(0.5-413) \\
76(76-286)\end{array}$ & $\begin{array}{l}136 \pm 154(0.5-413) \\
76(76-286)\end{array}$ & \begin{tabular}{|l|}
$173 \pm 97(45-338)$ \\
$150(101-258)$
\end{tabular} & $\begin{array}{l}173 \pm 97(45-338) \\
150(101-258)\end{array}$ & $\begin{array}{l}255 \pm 98(81-399) \\
233(202-342)\end{array}$ & $\begin{array}{l}255 \pm 98(81-399) \\
233(202-342)\end{array}$ & $\begin{array}{l}191 \pm 59(108-280) \\
167(148-245)\end{array}$ & $\begin{array}{l}191 \pm 59(108-280) \\
167(148-245)\end{array}$ \\
\hline $\begin{array}{l}\mathbf{H t}[\mathrm{L} / \mathrm{L}(\%)] \\
(0.37-0.55)\end{array}$ & $\begin{array}{l}0.50 \pm 0.05(0.43- \\
0.58) \\
0.50(0.47-0.53)\end{array}$ & $\begin{array}{l}50 \pm 5(43-58) \\
50(47-53)\end{array}$ & \begin{tabular}{|l}
$0.47 \pm 0.08(0.34-$ \\
$0.55)$ \\
$0.53(0.39-0.55)$
\end{tabular} & $\begin{array}{l}47 \pm 8(34-55) \\
53(39-55)\end{array}$ & \begin{tabular}{|l|}
$0.33 \pm 0.10(0.20-$ \\
$0.45)$ \\
$0.31(0.22-0.45)$ \\
\end{tabular} & $\begin{array}{l}33 \pm 10(20-45) \\
31(22-45)\end{array}$ & $\begin{array}{l}0.52 \pm 0.05(0.43- \\
0.58) \\
0.53(0.47-0.57)\end{array}$ & $\begin{array}{l}52 \pm 5(43-58) \\
53(47-57)\end{array}$ & $\begin{array}{l}0.44 \pm 0.08(0.33- \\
0.56) \\
0.46(0.37-0.50)\end{array}$ & $\begin{array}{l}44 \pm 8(33-56) \\
46(37-50)\end{array}$ \\
\hline$\underset{(6-15)}{\operatorname{WBC}}\left[\times 10^{9} \mathrm{~L}\left(\times 10^{3} / \mathrm{LL}\right)\right]$ & $\begin{array}{l}8.8 \pm 1.8(6.0-11.1) \\
8.8(6.8-10.6)\end{array}$ & $\begin{array}{l}8.8 \pm 1.8(6.0-11.1) \\
8.8(6.8-10.6)\end{array}$ & $\begin{array}{l}19.0 \pm 10.1(7.8- \\
39.1) \\
15.8(11.9-26.9) \\
\end{array}$ & \begin{tabular}{|l|}
$19.0 \pm 10.1(7.8-$ \\
$39.1)$ \\
$15.8(11.9-26.9)$ \\
\end{tabular} & \begin{tabular}{|l|}
$19.8 \pm 10.0(8.3-$ \\
$41.6)$ \\
$17.9(14.1-23.0)$ \\
\end{tabular} & $\begin{array}{l}19.8 \pm 10.0(8.3- \\
41.6) \\
17.9(14.1-23.0) \\
\end{array}$ & $\begin{array}{l}9.5 \pm 3.5(4.8-14.1) \\
8.5(6.3-13.0)\end{array}$ & \begin{tabular}{|l}
$9.5 \pm 3.5(4.8-14.1)$ \\
$8.5(6.3-13.0)$
\end{tabular} & $\begin{array}{l}8.8 \pm 5.5(1.1-16.7) \\
9.3(3.2-13.2)\end{array}$ & $\begin{array}{l}8.8 \pm 5.5(1.1-16.7) \\
9.3(3.2-13.2)\end{array}$ \\
\hline$\underset{(<30)}{\operatorname{CRP}[\mathrm{mg} / \mathrm{L}(\mathrm{mg} / \mathrm{dL})]}$ & $\begin{array}{l}5.7 \pm 1.9(5.1-11.1) \\
5.1(0)\end{array}$ & $\begin{array}{l}0.6 \pm 0.2(0.5-1.1) \\
0.5(0)\end{array}$ & $\begin{array}{l}12.8 \pm 15.7(5.1- \\
50.6) \\
5.1(5.1-13.2)\end{array}$ & \begin{tabular}{|l}
$1.3 \pm 1.6(0.5-5.1)$ \\
$0.5(0.5-1.3)$
\end{tabular} & \begin{tabular}{|l|}
$50.9 \pm 23.4(5.1-$ \\
$84.0)$ \\
$56.0(37.5-63.8)$
\end{tabular} & $\begin{array}{l}5.1 \pm 2.3(0.5-8.4) \\
5.6(3.8-6.4)\end{array}$ & $\begin{array}{l}5.1(0) \\
5.1(0)\end{array}$ & $\begin{array}{l}0.5(0) \\
0.5(0)\end{array}$ & \begin{tabular}{|l|}
$73.8 \pm 34.3(8.6-$ \\
$122.0)$ \\
$75.5(57.3-100.7)$
\end{tabular} & $\begin{array}{l}7.4 \pm 3.4(0.9-12.2) \\
7.6(5.7-10.1)\end{array}$ \\
\hline $\begin{array}{l}\text { R time (min) } \\
(3-9)\end{array}$ & $\begin{array}{l}8.2 \pm 3.2(4.2-13.8) \\
7.9(5.5-11.2)\end{array}$ & $\begin{array}{l}8.2 \pm 3.2(4.2-13.8) \\
7.9(5.5-11.2)\end{array}$ & $\begin{array}{l}33.4 \pm 38.3(4.1- \\
119.9) \\
21.9(8.6-53.0) \\
\end{array}$ & \begin{tabular}{|l|}
$33.4 \pm 38.3(4.1-$ \\
$119.9)$ \\
$21.9(8.6-53.0)$ \\
\end{tabular} & \begin{tabular}{|l|}
$22.3 \pm 40.0(2.2-$ \\
$119.9)$ \\
$6.6(3.6-19.3)$ \\
\end{tabular} & $\begin{array}{l}22.3 \pm 40.0(2.2- \\
119.9) \\
6.6(3.6-19.3) \\
\end{array}$ & $\begin{array}{l}7.8 \pm 4.6(0.2-15.8) \\
7.2(5.7-10.9)\end{array}$ & $\begin{array}{l}7.8 \pm 4.6(0.2-15.8) \\
7.2(5.7-10.9)\end{array}$ & \begin{tabular}{|l|}
$9.3 \pm 3.1(3.8-13.9)$ \\
$9.1(7.6-12.2)$ \\
\end{tabular} & $\begin{array}{l}9.3 \pm 3.1(3.8-13.9) \\
9.1(7.6-12.2)\end{array}$ \\
\hline $\begin{array}{l}\text { K time (min) } \\
(2-8)\end{array}$ & $\begin{array}{l}3.4 \pm 1.3(1.4-5.4) \\
3.8(2.2-4.3)\end{array}$ & $\begin{array}{l}3.4 \pm 1.3(1.4-5.4) \\
3.8(2.2-4.3)\end{array}$ & $\begin{array}{l}11.0 \pm 18.8(0-49.1) \\
3.9(1.2-18.1)\end{array}$ & $\begin{array}{l}11.0 \pm 18.8(0-49.1) \\
3.9(1.2-18.1)\end{array}$ & \begin{tabular}{|l|}
$4.6 \pm 5.5(0-16.2)$ \\
$2.4(0.9-8.0)$
\end{tabular} & $\begin{array}{l}4.6 \pm 5.5(0-16.2) \\
2.4(0.9-8.0)\end{array}$ & $\begin{array}{l}3.7 \pm 1.5(2.0-6.63) \\
3.3(2.5-5.2)\end{array}$ & $\begin{array}{l}3.7 \pm 1.5(2.0-6.3) \\
3.3(2.5-5.2)\end{array}$ & $\begin{array}{l}3.4 \pm 1.1(1.9-5.2) \\
3.4(2.5-4.3)\end{array}$ & $\begin{array}{l}3.4 \pm 1.1(1.9-5.2) \\
3.4(2.5-4.3)\end{array}$ \\
\hline $\begin{array}{l}\text { Angle (degrees) } \\
\text { (27-59) }\end{array}$ & $\begin{array}{l}46.5 \pm 13.3(26.3- \\
70.2) \\
45.5(36.5-58.4)\end{array}$ & $\begin{array}{l}46.5 \pm 13.3(26.3- \\
70.2) \\
45.5(36.5-58.4) \\
\end{array}$ & $\begin{array}{l}21.2 \pm 25.0(0-67.8) \\
4.8(0.7-42.4)\end{array}$ & $\begin{array}{l}21.2 \pm 25.0(0-67.8) \\
4.8(0.7-42.4)\end{array}$ & \begin{tabular}{|l}
$42.1 \pm 29.4(0-78.9)$ \\
$42.1(17.0-71.3)$
\end{tabular} & $\begin{array}{l}42.1 \pm 29.4(0-78.9) \\
42.1(17.0-71.3)\end{array}$ & $\begin{array}{l}41.1 \pm 12.1(25.1- \\
58.5) \\
37.4(30.5-52.3) \\
\end{array}$ & \begin{tabular}{|l|}
$41.1 \pm 12.1(25.1-$ \\
$58.5)$ \\
$37.4(30.5-52.3)$ \\
\end{tabular} & $\begin{array}{l}50.2 \pm 8.3(41.7- \\
66.8) \\
46.5(44.2-57.1)\end{array}$ & $\begin{array}{l}50.2 \pm 8.3(41.7- \\
66.8) \\
46.5(44.2-57.1)\end{array}$ \\
\hline$\underset{(39-59)}{\text { MA (mm) }}$ & $\begin{array}{l}58.8 \pm 6.4(45.9- \\
66.2) \\
60.1(53.6-63.8) \\
\end{array}$ & $\begin{array}{l}58.8 \pm 6.4(45.9- \\
66.2) \\
60.1(53.6-63.8) \\
\end{array}$ & $\begin{array}{l}31.2 \pm 29.8(0-74.0) \\
12.1(5.8-61.0)\end{array}$ & $\begin{array}{l}31.2 \pm 29.8(0-74.0) \\
12.1(5.8-61.0)\end{array}$ & $\begin{array}{l}60.9 \pm 26.3(0-82.1) \\
70.3(54.0-73.9)\end{array}$ & $\begin{array}{l}60.9 \pm 26.3(0-82.1) \\
70.3(54.0-73.9)\end{array}$ & $\begin{array}{l}54.2 \pm 21.0(2.4- \\
70.2) \\
59.9(49.3-68.8)\end{array}$ & \begin{tabular}{|l|}
$54.2 \pm 21.0(2.4-$ \\
$70.2)$ \\
$59.9(49.3-68.8)$ \\
\end{tabular} & $\begin{array}{l}71.7 \pm 5.9(64.6- \\
83.5) \\
70.5(67.4-75.0)\end{array}$ & $\begin{array}{l}71.7 \pm 5.9(64.6- \\
83.5) \\
70.5(67.4-75.0) \\
\end{array}$ \\
\hline $\begin{array}{l}\text { G value }\left(\mathrm{dyn} / \mathrm{cm}^{2}\right) \\
(3.2 \mathrm{~K}-7.2 \mathrm{~K})\end{array}$ & $\begin{array}{l}7.4 \pm 1.8(4.2-9.8) \\
7.6(5.8-8.9)\end{array}$ & $\begin{array}{l}7.4 \pm 1.8(4.2-9.8) \\
7.6(5.8-8.9)\end{array}$ & $\begin{array}{l}4.1 \pm 5.0(0-14.2) \\
0.7(0.3-7.8)\end{array}$ & \begin{tabular}{|l|}
$4.1 \pm 5.0(0-14.2)$ \\
$0.7(0.3-7.8)$
\end{tabular} & \begin{tabular}{|l|}
$11.3 \pm 6.8(0-23.0)$ \\
$11.9(6.3-14-2)$
\end{tabular} & $\begin{array}{l}11.3 \pm 6.8(0-23.0) \\
11.9(6.3-14.2)\end{array}$ & $\begin{array}{l}7.4 \pm 3.8(0.1-111.8) \\
7.5(4.9-11.1)\end{array}$ & $\begin{array}{l}7.4 \pm 3.8(0.1-11.8) \\
7.5(4.9-11.1)\end{array}$ & $\begin{array}{l}13.6 \pm 5.1(9.1-25.4) \\
12.0(10.4-15.4)\end{array}$ & $\begin{array}{l}13.6 \pm 5.1(9.1-25.4) \\
12.0(10.4-15.4)\end{array}$ \\
\hline $\begin{array}{l}\text { AT }(\%) \\
(>80)\end{array}$ & $\begin{array}{l}122 \pm 14(107-144) \\
117(110-138)\end{array}$ & $\begin{array}{l}122 \pm 14(107-144) \\
117(110-138)\end{array}$ & $\begin{array}{l}97 \pm 21(66-138) \\
94(83-108)\end{array}$ & \begin{tabular}{|l|}
$97 \pm 21(66-138)$ \\
$94(83-108)$
\end{tabular} & \begin{tabular}{|l|}
$92 \pm 28(59-139)$ \\
$91(64-112)$
\end{tabular} & $\begin{array}{l}92 \pm 28(59-139) \\
91(64-112)\end{array}$ & $\begin{array}{l}99 \pm 12(73-117) \\
100(94-106)\end{array}$ & $\begin{array}{l}99 \pm 12(73-117) \\
100(94-106)\end{array}$ & $\begin{array}{l}89 \pm 15(68-107) \\
87(75-106)\end{array}$ & $\begin{array}{l}89 \pm 15(68-107) \\
87(75-106)\end{array}$ \\
\hline $\begin{array}{l}\text { Fib }[g / L(m g / d L)] \\
(2-4)\end{array}$ & $\begin{array}{l}2.5 \pm 0.5(1.7-3.4) \\
2.5(2.1-2.8)\end{array}$ & $\begin{array}{l}250 \pm 50(170-340) \\
250(210-280)\end{array}$ & $\begin{array}{l}2.9 \pm 0.5(2.1-3.7 .7) \\
3.0(2.6-3.2)\end{array}$ & \begin{tabular}{|l|}
$290 \pm 50(210-370)$ \\
$300(260-320)$
\end{tabular} & \begin{tabular}{|l|}
$3.8 \pm 1.6(0.6-5.9)$ \\
$3.9(3.2-5.2)$
\end{tabular} & $\begin{array}{l}380 \pm 160(60-590) \\
390(320-520)\end{array}$ & $\begin{array}{l}2.8 \pm 1.5(1.0-6.3) \\
2.3(2.1-3.2)\end{array}$ & $\begin{array}{l}280 \pm 150(100-630) \\
230(210-320)\end{array}$ & $\begin{array}{l}5.1 \pm 1.2(3.0-7.2) \\
5.1(0.2-5.2)\end{array}$ & $\begin{array}{l}510 \pm 120(300-720) \\
510(20-520)\end{array}$ \\
\hline PT $(\mathrm{sec})$ & $\begin{array}{l}6.5 \pm 1.6(5.0-10.7) \\
6.4(5.5-6.7)\end{array}$ & $\begin{array}{l}6.5 \pm 1.6(5.0-10.7) \\
6.4(5.5-6.7)\end{array}$ & $\begin{array}{l}7.6 \pm 1.3(6.6-10.4) \\
7.0(6.8-8.5)\end{array}$ & $\begin{array}{l}7.6 \pm 1.3(6.6-10.4) \\
7.0(6.8-8.5)\end{array}$ & \begin{tabular}{|l|}
$7.2 \pm 0.8(6.4-8.83)$ \\
$6.9(6.5-8.1)$
\end{tabular} & $\begin{array}{l}7.2 \pm 0.8(6.4-8.3) \\
6.9(6.5-8.1)\end{array}$ & $\begin{array}{l}7.8 \pm 1.5(6.4-11.0) \\
7.3(7.0-8.6)\end{array}$ & $\begin{array}{l}7.8 \pm 1.5(6.4-11.0) \\
7.3(7.0-8.6)\end{array}$ & $\begin{array}{l}7.2 \pm 0.8(6.2-8.6) \\
7.3(6.6-7.8)\end{array}$ & $\begin{array}{l}7.2 \pm 0.8(6.2-8.6) \\
7.3(6.6-7.8)\end{array}$ \\
\hline aPTT (sec) & $\begin{array}{l}11.1 \pm 0.4(10.3- \\
11.6) \\
11.2(11.0-11.4)\end{array}$ & $\begin{array}{l}11.1 \pm 0.4(10.3- \\
11.6) \\
11.2(11.0-11.4)\end{array}$ & $\begin{array}{l}11.9 \pm 1.3(10.7- \\
14.2) \\
11.6(11.0-13.1)\end{array}$ & \begin{tabular}{|l|}
$11.9 \pm 1.3(10.7-$ \\
$14.2)$ \\
$11.6(11.0-13.1)$
\end{tabular} & \begin{tabular}{|l|}
$12.3 \pm 1.5(10.6-$ \\
$15.5)$ \\
$12.0(11.3-13.0)$
\end{tabular} & $\begin{array}{l}12.3 \pm 1.5(10.6- \\
15.5) \\
12.0(11.3-13.0)\end{array}$ & $\begin{array}{l}12.3 \pm 1.3(10.4- \\
14.2) \\
12.2(11.0-13.3)\end{array}$ & \begin{tabular}{|l|}
$12.3 \pm 1.3(10.4-$ \\
$14.2)$ \\
$12.2(11.0-13.3)$
\end{tabular} & $\begin{array}{l}12.0 \pm 1.6(10.0- \\
14.3) \\
12.0(10.4-13.5)\end{array}$ & $\begin{array}{l}12.0 \pm 1.6(10.0- \\
14.3) \\
12.0(10.4-13.5)\end{array}$ \\
\hline
\end{tabular}




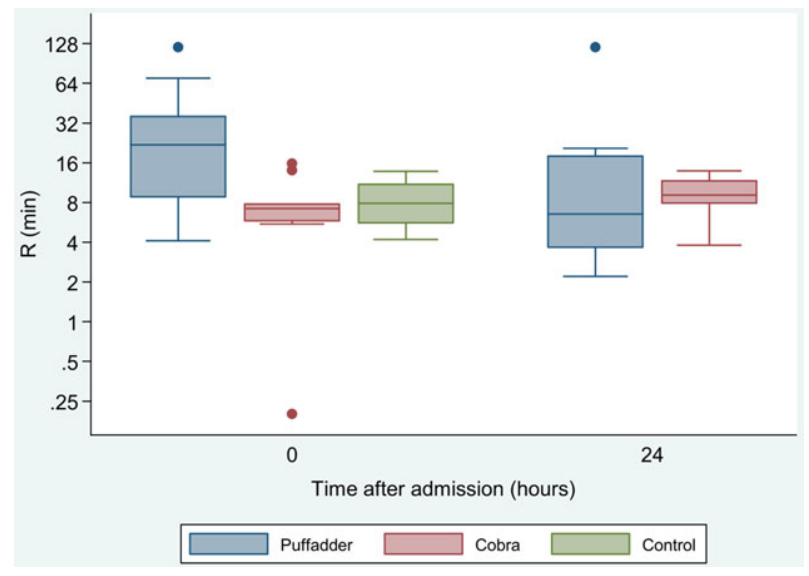

Figure 1: $R$ time for puffadder, snouted cobra, and control groups at admission and 24 hours post envenomation.

$(1 / 9)$, whole blood $(1 / 9)$, and polyvalent antivenom (2/9); and for the cobra group included polyvalent antivenom (8/9). Two dogs in the puffadder group died within 24 hours of admission and 1 dog in the cobra group was euthanized 72 hours post presentation due to a poor prognosis. The 2 dogs $(<10 \mathrm{~kg})$ that died after puffadder envenoming in this study did not show any antemortem macroscopic evidence of bleeding, except mild-to-moderate soft tissue swelling and oozing at the envenomation sites. Neither dog was treated with polyvalent antivenom or any blood products. However, postmortem examination demonstrated multiple hemorrhages in the subcutis and at the envenomation sites (musculature) of both dogs. Additionally, there were several small, paintbrush hemorrhages in the endocardium of the left ventricle in the $1 \mathrm{dog}$ and moderate enterorrhagia in the small intestine and cecum was noted in the other fatality.

Comparison of hemostatic variables, and associated blood parameters, between groups at presentation

Table 1 contains a summary of all the variables for the 3 groups at presentation and at 24 hours. The most significant result was the mean $R$ time on presentation, which was significantly prolonged in the puffadder group compared to the cobra $(P=0.01)$ and control groups $(P=0.05)$; however, there was no significant difference between the cobra and control groups (Figure 1). None of the other TEG variables were significantly different among groups. Plt was significantly lower in the puffadder compared to the cobra $(P=0.04)$ and control groups $(P=0.001)$; however, there was no significant difference between the cobra versus control groups (Figure 2). HCT was not significantly different among groups. WBC was significantly higher in the puffadder compared to the co-

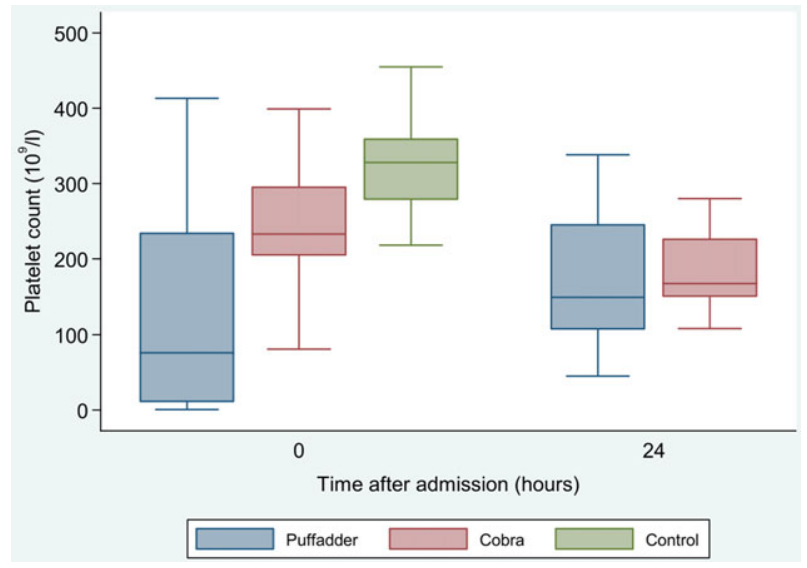

Figure 2: Platelet count for puffadder, snouted cobra, and control groups at admission and 24 hours post envenomation.

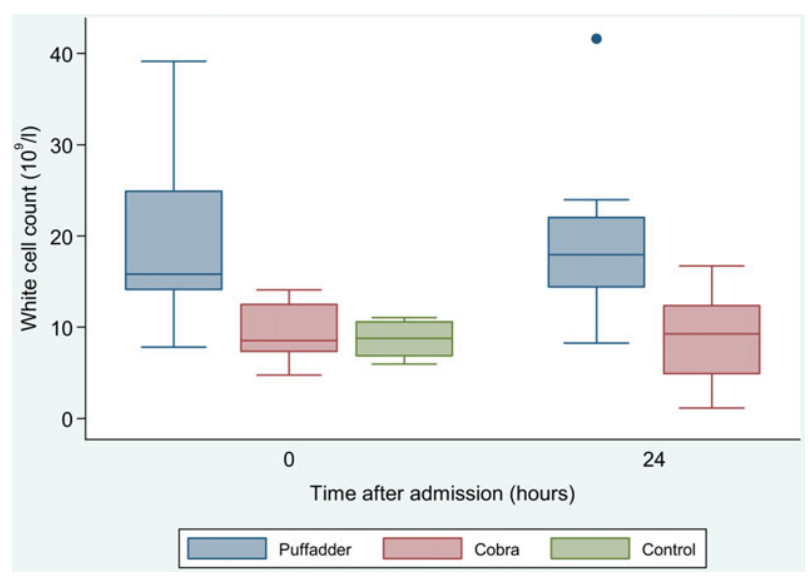

Figure 3: White cell count for puffadder, snouted cobra, and control groups at admission and 24 hours post envenomation.

bra $(P=0.003)$ and control groups $(P=0.001)$; however, there was no significant difference between the cobra and control groups (Figure 3). CRP concentration was not significantly different among groups (Figure 4). Fib concentration was not significantly different among groups. AT activity was significantly decreased in the puffadder $(P=0.002)$ and cobra groups $(P=0.004)$ compared to the control group; however, there was no significant difference between the puffadder and cobra groups (Figure 5). PT was significantly prolonged in the cobra compared to the control group $(P=0.03)$; however, there was no significant difference between the puffadder versus cobra or control groups. aPTT was significantly prolonged in the cobra compared to the control group $(P=0.03)$; however, there was no significant difference between the puffadder versus cobra or control groups. 


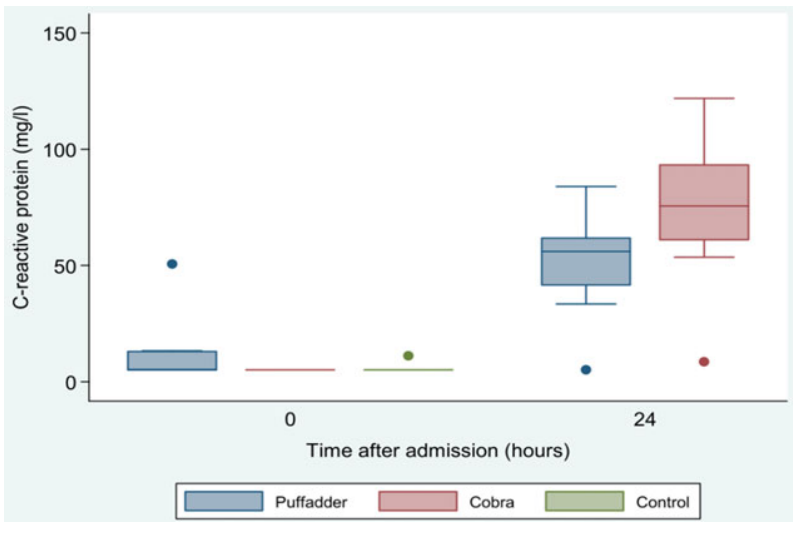

Figure 4: C-reactive protein for puffadder, snouted cobra, and control groups at admission and 24 hours post envenomation.

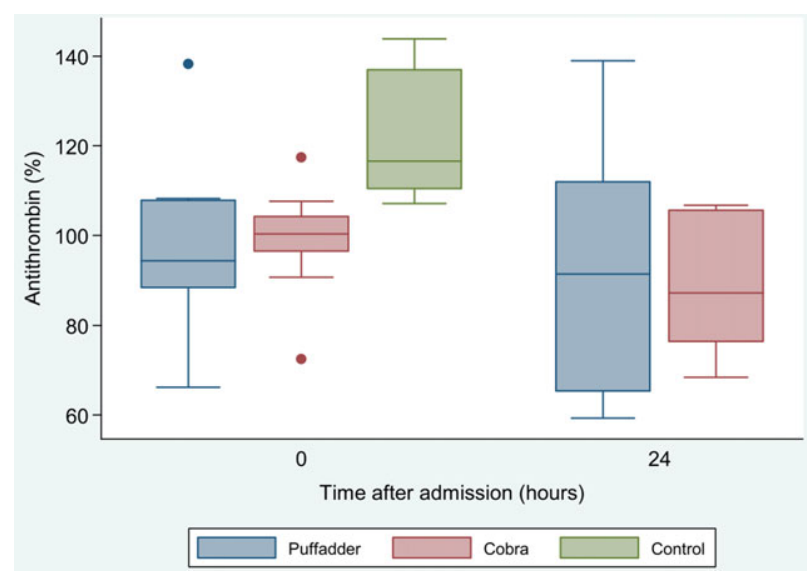

Figure 5: Antithrombin for puffadder, snouted cobra, and control groups at admission and 24 hours post envenomation.

Change over time in hemostatic variables, and associated blood parameters, for the puffadder and cobra groups, and differences between groups at 24 hours post envenomation

Of the TEG variables, the MA and $G$ were significantly increased in the puffadder group at 24 hours when compared to the values at presentation $(P=0.05$ for both); however, there were no significant changes in these variables for the cobra group. There were no significant changes over time for $R, K$, or angle in either group. There were no significant changes in Plt, but HCT was significantly lower at 24 hours in both groups $(P<0.001$ and $P=0.02$, respectively). There were no significant changes for WBC; however, there were significant increases in CRP concentration in both groups $(P=0.04$ and $P=0.001$, respectively), as well as Fib concentration for the cobra group $(P<0.001)$. There were no significant changes for AT activity, PT, or aPTT. Treatment with blood products had no effect on any of these variables; however, dogs treated with polyvalent antivenom had a significantly lower AT activity $(P=0.05)$ and prolonged aPTT $(P=0.01)$ in both the puffadder and cobra groups; however, the values for both variables were still within the normal reference limits. When variables were compared between groups at 24 hours post envenomation, HCT was significantly decreased and WBC increased $(P=0.01$ for both) in the puffadder compared to the cobra group. For all other variables, there were no significant differences between the groups.

\section{Discussion}

This study demonstrated that dogs envenomed by puffadders were more hypocoagulable at presentation, characterized by a significantly prolonged clot initiation time $(R)$, compared to dogs envenomed by snouted cobras and healthy controls. This finding was surprising since envenomations by puffadders are considered to be extremely cytotoxic in nature and therefore a hypercoagulable status was expected, secondary to inflammation-triggered activation of coagulation. ${ }^{4,11-13,23,27}$ The hypocoagulable status was transient and 24 hours post envenomation the prolonged $R$ time had normalized in most of the dogs without any specific treatment administered (eg, polyvalent antivenom, fresh frozen plasma, stored whole blood). This could be a venom-induced phenomenon and could potentially occur due to abnormal thrombin production or a direct effect on certain coagulation factors. The TEG variables $K$, angle, and MA of the puffadder group were not significantly different compared to the cobra and control groups at presentation. Visual appreciation of the thromboelastograms of puffadder-envenomed dogs, however, clearly demonstrated hypocoagulability together with prolonged clot initiation compared to the other 2 groups (Figures 6 and 7). The lack of significance is most likely due to the fact that these variables were adjusted for the effect that Plt and Fib concentration can have on them. The small sample size most likely also affected the power of the study and contributed to this loss of significance of the TEG variables between groups.

A prolonged $R$ time correlates with delayed initiation of hemostasis ${ }^{14,28}$ and supports the theory of a venominduced phenomenon, refractory to treatment with blood components. Snake venoms are known to contain proteins that can activate or prevent hemostasis. , $^{89,30}$ Examples of anticoagulant venom components include phospholipases, fibrinogenolytic enzymes, and proteolytic enzymes that can degrade coagulation factors. ${ }^{31}$ The coagulopathy and hemorrhage that results from envenomation by snakes belonging to the family Viperidae (B. gabonica, B. arietans) in human patients is well described. $8,22,32$ The venom of the gaboon adder (B. gabonica) contains components that interfere with 


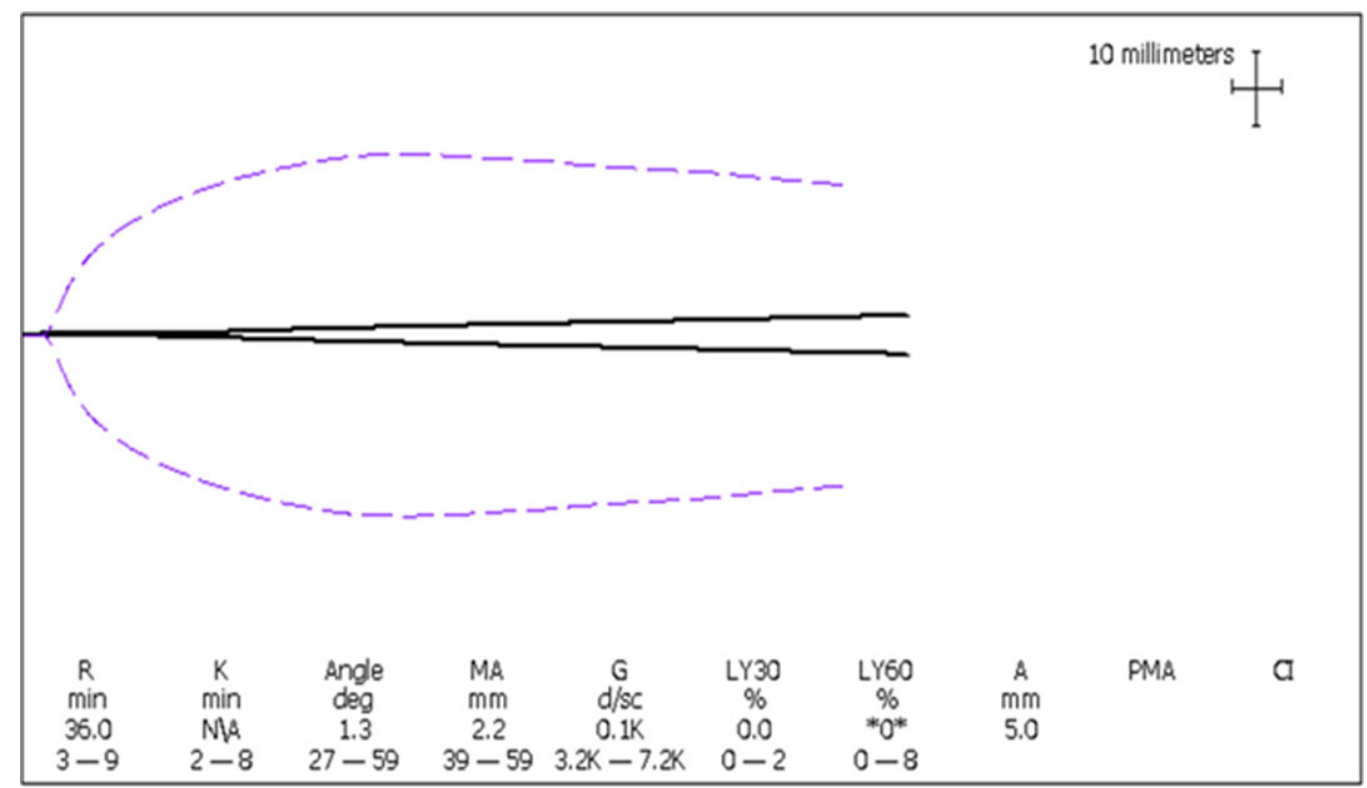

Figure 6: TEG of a dog in the puffadder group at admission demonstrating a hypocoagulable tracing. Dashed line indicates a normal tracing.

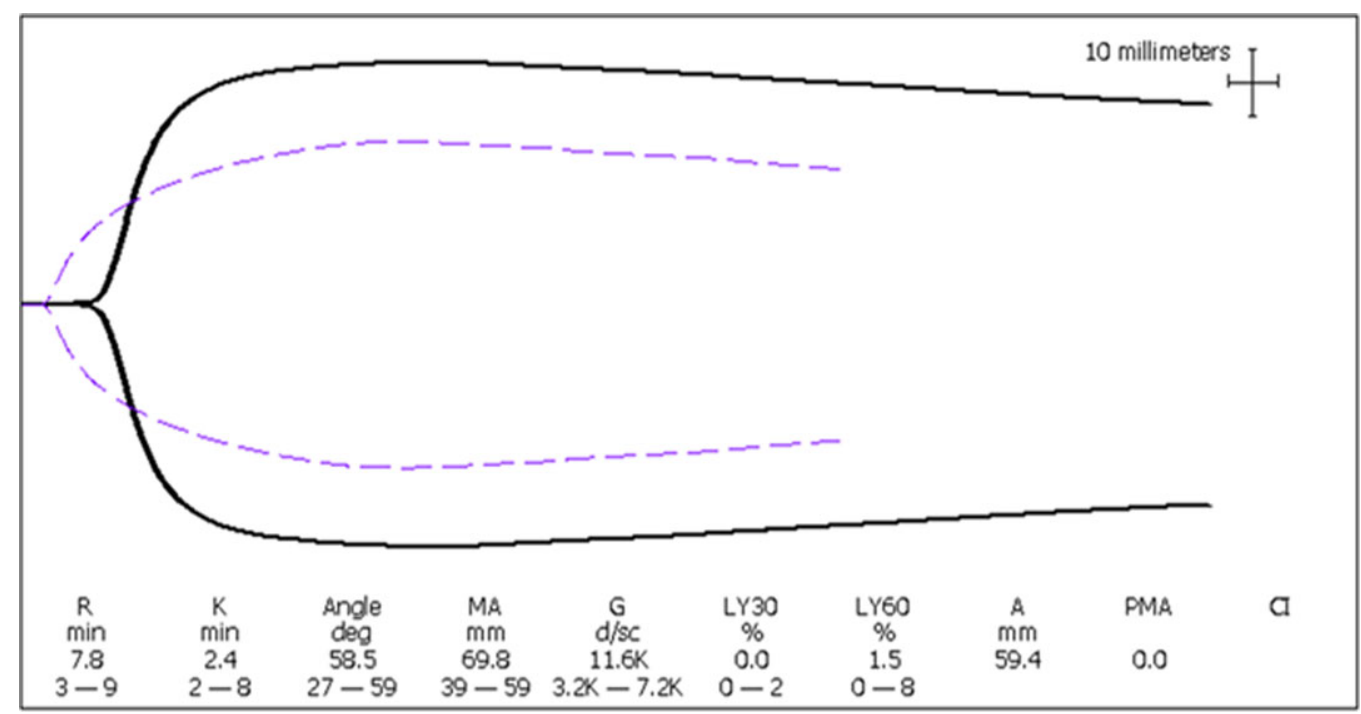

Figure 7: TEG of a dog in the cobra group at admission demonstrating a hypercoagulable tracing. Dashed line indicates a normal tracing.

platelet aggregation, as well as the blood thromboplastin system, resulting in defective thrombin generation, defective prothrombin consumption, inhibition of thromboplastin formation, and accelerated loss of formed thromboplastin. ${ }^{32}$ Clot formation is also impaired through direct proteolytic action on Fib, releasing soluble Fib breakdown products, with possibly defective fibrin polymerization, resulting in a prolonged thrombin clotting time. ${ }^{32}$ The effects of puffadder venom on coagulation in baboons also demonstrated fibrinolysis and fibrinogenolysis and appear dose related, with evidence of consumption of blood coagulation factors and severe thrombocytopenia. ${ }^{33}$ In addition, normal human platelets were shown to be extremely sensitive to small doses of venom in vitro, with irreversible aggregation occurring very rapidly, probably through the action of adenosine diphosphate. ${ }^{33}$

Significant thrombocytopenia was noted on presentation in the puffadder group compared to both cobra and control groups, which is similar to previous reports in 
dogs ${ }^{23}$ and baboons. ${ }^{33}$ Venom-induced thrombocytopenia has been described in puffadder victims. ${ }^{33}$ Snake venom is able to target platelets by either inhibiting (thereby reducing their effectiveness in hemostasis), or promoting aggregation (contributing toward pathologic sequelae). ${ }^{8}$ Both platelet aggregators (bitiscetin) and inhibitors (bitistatin) have been isolated from the venom of $B$. arietans. Bitiscetin, a disulphide-linked heterodimer, is a C-type lectin-like protein that binds to the $\mathrm{A}_{1}$ domain of Von Willebrand's factor and induces platelet aggregation through binding of platelet membrane glycoprotein $\mathrm{Ib}^{31,34}$ Bitistatin, on the other hand, is a disintegrin (receptor antagonist) that binds to integrin Ilb-IIIa receptors on the platelet surface, thereby blocking Fib binding to the receptor-glycoprotein complex on activated platelets. ${ }^{35}$ Platelet aggregation induced by action of adenosine diphosphate, thrombin, platelet-activating factor, and collagen therefore becomes inhibited. ${ }^{8,36}$ This venom effect may be an explanation for the low MA observed in most of the dogs envenomed by puffadder. None of the cobra-envenomed dogs demonstrated signs of thrombocytopenia.

Other hematological findings at presentation included a normal HCT and leukocytosis in the puffadder group. At 24 hours, the HCT had decreased, but still remained within the normal reference interval. This finding is most likely attributed to acute hemorrhage that occurs at the envenomation site, ${ }^{4,23}$ with resultant fluid shifts due to capillary leakage. ${ }^{6}$ Puffadders are known for their highly potent hemorrhagic and cytotoxic venom, ${ }^{22,27}$ and patients usually develop marked swelling at the envenomation site that is progressive for up to 72 hours. ${ }^{4}$ The HCT also decreased in the cobra group at 24 hours, but remained well within the reference interval. Administration of IV fluids will also result in hemodilution, which likely lowered HCT in both puffadder and cobra groups.

A marked leukocytosis was present in the puffadderenvenomed dogs at all times of blood sampling and has also been described previously. ${ }^{23}$ The leukocytosis is likely induced by cytokines as a result of acute inflammation triggered by cellular damage at the envenomation site, but could also occur due to a stress or pain response. ${ }^{3,6,23}$ Severe tissue damage at the envenomation site is characteristic of envenoming by viperid and some elapid snakes. A complex of pathological effects result, such as skin and muscle necrosis, blistering, hemorrhage, lymphatic vessel damage, edema, and extracellular matrix degradation. Many venom components are responsible for these effects and include phospholipase $\mathrm{A}_{2}$ and snake venom metalloproteinases. ${ }^{10}$ Inflammation is a key feature in snake envenomation as can also be demonstrated by the significant increases in CRP and Fib concentrations during the first 24 hours in this study. Only 3 dogs (3/18) demonstrated an increased CRP concentration at presentation; all 3 were envenomed by puffadders. At 24 hours, the majority of dogs (apart from 2) had increased CRP concentrations, suggesting continuing inflammation, which is likely the largest contributing factor of progressive hypercoagulability in both puffadder- and cobra-envenomed dogs.

Of the traditional plasma-based coagulation assays, AT activity, PT, and aPTT were found to be significantly different among the groups. Although AT activity was decreased at presentation in the puffadder and cobra groups compared to the controls, it remained within the normal reference limits in most patients (activity $>80 \%$ ). It would seem that both types of snakes initiate nonspecific coagulation, with resultant consumption of AT. This effect was also seen 24 hours post envenomation, where AT activity was decreased even more, but still remained within the normal reference limits. PT and aPTT were both significantly prolonged in the cobra-envenomed dogs compared to the controls; however, the values also remained within normal reference limits. The clinical relevance of the significant differences among groups for AT activity, PT, and PTT is therefore questionable because the differences were small, with overlap between the groups.

Limitations to this study included a small sample size and therefore a high probability of type II error. The statistical power of the study may also have been reduced due to the necessity for adjusting for covariates, such as the low Plt and high Fib concentration that have been shown to affect certain TEG variables, as well as the disease-specific treatments provided (polyvalent antivenom, blood products). Time from envenomation to presentation could not be standardized. In an attempt to overcome this limitation, the authors chose to include only cases that presented within 6 hours post envenoming. Dogs were therefore at differing stages of hemostasis when presented, which could have influenced the results. The large variation in body mass between the cases may have affected the venom-to-body mass ratio, which may also have affected the results. Due to budget constraints, blood-borne parasites, such as $B$. rossi and $E$. canis, could not be definitively excluded by polymerase chain reaction testing.

\section{Conclusions}

Hemostatic changes occur in dogs envenomed by both puffadder and snouted cobra. The hypocoagulable changes in dogs envenomed by puffadders are likely venom induced, dose-dependent, and transient; most puffadder victims reverted to a hypercoagulable state within 24 hours post envenoming due to likely on-going local and systemic inflammation. We postulate that the 
hypocoagulable thromboelastogram seen with puffadder envenoming at presentation could be due to abnormal thrombin production, or a direct effect on coagulation factors. Snouted cobra-envenomed dogs were found to be hypercoagulable at presentation. This hypercoagulability appears to be progressive, as follow-up thromboelastograms 24 hours post envenomation were more hypercoagulable than at presentation. These dogs should be monitored for thrombosis that may result in pulmonary thromboembolism or multiple organ damage. However, the use of anticoagulants such as heparin is reported to be contra-indicated because most venominduced coagulopathies have been shown to be resistant to heparin. ${ }^{37}$ The presence of fibrin degradation products in the victim may also contribute to the anticoagulant effect and spontaneous bleeding may result. 8,38

TEG proved to be a more sensitive tool in detecting abnormal hemostatic status in envenomed victims compared to traditional coagulation assays. An abnormal thromboelastogram in a puffadder-envenomed dog should prompt the clinician to administer appropriate antivenom and to monitor for a bleeding diathesis. Unfortunately, the use of TEG is currently limited to educational institutions due to expensive laboratory equipment.

\section{Acknowledgments}

The supporting staff in ICU and the technical staff of the Clinical Pathology Laboratory are thanked for their assistance with collection of samples and performing the assays. Funding was obtained from the Faculty Research Committee and the CACS Departmental Research Fund as well as the South African National Research Foundation.

\section{Footnotes}

a SAIMR Polyvalent Snake Antivenom, South African Vaccine Producers, Sandringham, RSA.

b BD Vacutainer, Becton, Dickinson and Company, NJ.

Forma Scientific $-86^{\circ} \mathrm{C}$ freezer, Labotec, Midrand, RSA.

d ADVIA 2120, Siemens, Marburg, Germany.

e ST art 4 analyzer, Diagnostica Stago, Roche, Asnières-sur-Seine, France.

Cobas Integra 400 Plus analyzer, Roche, Basel, Switzerland.

g TEG 5000 Thromboelastograph $\AA$ Hemostasis System, Hemoscope, Niles, IL.

$\mathrm{h}$ Dade Innovin, Siemens.

i NCSS 2007, Kaysville, UT.

j Stata 12.1, StataCorp, College Station, TX.

\section{References}

1. Corbett SW, Anderson B, Nelson B, et al. Most lay people can correctly identify indigenous venomous snakes. Am J Emerg Med 2005; 23(6):759-762.

2. Heller J, Bosward KL, Hodgson JL, et al. Snake envenomation in dogs in New South Wales. Aust Vet J 2005; 83(5):286-292.
3. Heller J, Mellor DJ, Hodgson JL, et al. Elapid snake envenomation in dogs in New South Wales: a review. Aust Vet J 2007; 85(11):469-479.

4. Leisewitz AL, Blaylock RS, Kettner F, et al. The diagnosis and management of snakebite in dogs-a Southern African perspective. J S Afr Vet Assoc 2004; 75(1):7-13.

5. Juárez $\mathrm{P}$, Wagstaff SC, Oliver J, et al. Molecular cloning of disintegrin-like transcript BA-5A from a Bitis arietans venom gland cDNA library: a putative intermediate in the evolution of the longchain disintegrin bitistatin. J Mol Evol 2006; 63:142-152.

6. Goddard A, Schoeman JP, Leisewitz AL, et al. Clinicopathologic abnormalities associated with snake envenomation in domestic animals. Vet Clin Pathol 2011; 40(3):282-292.

7. Gilliam LL, Brunker J. North American snake envenomation in the dog and cat. Vet Clin North Am Small Anim Pract 2011; 41:12391259.

8. White J. Snake venoms and coagulopathy. Toxicon 2005; 45(8):951967.

9. Moreira V, Dos-Santos MC, Nascimento NG, et al. Local inflammatory events induced by Bothropsatrox snake venom and the release of distinct classes of inflammatory mediators. Toxicon 2012; 60(1):1220.

10. Teixeira C, Cury Y, Moreira V, et al. Inflammation induced by Bothrops asper venom. Toxicon 2009; 54(7):988-997.

11. Chu AJ. Tissue factor, blood coagulation and beyond: an overview. Int J Inflam 2011; doi: 10.4061/2011/367284 (Epub September 20, 2011).

12. Levi M, Van der Poll T. Two-way interactions between inflammation and coagulation. Trends Cardiovasc Med 2005; 15(7):254-259.

13. Levi M, Keller TT, Van Gorp E, et al. Infection and inflammation and the coagulation system. Cardiovasc Res 2003; 60(1):26-39.

14. Wiinberg B, Jensen AL, Rojkjaer R, et al. Validation of human recombinant tissue factor-activated thromboelastography on citrated whole blood from clinically healthy dogs. Vet Clin Pathol 2005; 34(4):389-393.

15. Wiinberg B, Jensen AL, Johansson PI, et al. Thromboelastographic evaluation of hemostatic function in dogs with disseminated intravascular coagulation. J Vet Intern Med 2008; 22(2):357-365.

16. Fenty RK, Delaforcade AM, Shaw SE, et al. Identification of hypercoagulability in dogs with primary immune-mediated hemolytic anemia by means of thromboelastography. J Am Vet Med Assoc 2011; 238(4):463-467.

17. Goggs R, Wiinberg B, Kjelgaard-Hansen M, et al. Serial assessment of the coagulation status of dogs with immune-mediated haemolytic anaemia using thromboelastography. Vet J 2012; 191(3):347-353.

18. Goodwin LV, Goggs R, Chan DL, et al. Hypercoagulability in dogs with protein-losing enteropathy. J Vet Intern Med 2011; 25(2):273277.

19. Klose TC, Creevy KE, Brainard BM. Evaluation of coagulation status in dogs with naturally occurring canine hyperadrenocorticism. J Vet Emerg Crit Care 2011; 21(6):625-632.

20. Hadley GP, McGarr P, Mars M. The role of thromboelastography in the management of children with snake-bite in southern Africa. Trans R Soc Trop Med Hyg 1999; 93(2):177-179.

21. Marais J. Snakes \& Snake Bite in Southern Africa. Cape Town: Struik Publishers (Pty) Ltd; 1999, pp. 88.

22. Currier RB, Harrison RA, Rowley PD, et al. Intra-specific variation in venom of the African puff adder (Bitis arietans): differential expression and activity of snake venom metalloproteinases (SVMPs). Toxicon 2010; 55:864-873.

23. Lobetti RG, Joubert K. Retrospective study of snake envenomation in 155 dogs from the Onderstepoort area of South Africa. J S Afr Vet Assoc 2004; 75(4):169-172.

24. Bateman S, Mathews K, Abrams-Ogg A, et al. Evaluation of the effect of storage at $-70^{\circ} \mathrm{C}$ for six months on hemostatic function testing in dogs. Can J Vet Res 1999; 63(3):216-220.

25. Woodhams B, Girardot O, Blanco MJ, et al. Stability of coagulation proteins in frozen plasma. Blood Coagul Fibrinolysis 2001; 12(4):229-236.

26. Kjelgaard-Hansen M, Jensen AL, Kristensen AT. Evaluation of a commerically available human C-reactive protein (CRP) turbidometric immunoassay for determination of canine serum CRP concentration. Vet Clin Pathol 2003; 32(2):81-87. 
27. World Health Organization (July 2010). Guidelines for the Prevention and Clinical Management of Snakebite in Africa. Available at: http://www.afro.who.int/en/clusters-a-programmes / hss/essential-medicines/highlights/2731-guidelines-for-theprevention-and-clinical-management-of-snakebite-in-africa.html. Accessed June 29, 2012.

28. Kol A, Borjesson DL. Application of thromboelastography/ thromboelastometry to veterinary medicine. Vet Clin Pathol 2010; 39(4):405-416.

29. Marsh N, Williams V. Practical applications of snake venom toxins in haemostasis. Toxicon 2005; 45(8):1171-1181.

30. Tanos PP, Isbister GK, Lalloo DG, et al. A model for venom-induced consumptive coagulopathy in snake bite. Toxicon 2008; 52(7):769780 .

31. Morita T. Structures and functions of snake venom CLPs (C-type lectin-like proteins) with anticoagulant-, procoagulantand platelet-modulating activities. Toxicon 2005; 45(8):1099-1114

32. Forbes CD, Turpie AGG, Ferguson JC, et al. Effect of gaboon viper (Bitis gabonica) venom on blood coagulation, platelets and the fibrinolytic enzyme system. J Clin Pathol 1969; 22:312316.

33. Brink S, Steytler JG. Effects of puff-adder venom on coagulation, fibrinolysis and platelet aggregation in the baboon. S Afr Med J 1974; 48:1205-1213.

34. Lu Q, Navdaev A, Clemetson JM, et al. Snake venom C-type lectins interacting with platelet receptors. Structure-function relationships and effects on haemostasis. Toxicon 2005; 45(8):1089-1098.

35. Calvete JJ, Marcinkiewicz C, Monleon D, et al. Snake venom disintegrins: evolution of structure and function. Toxicon 2005; 45(8):10631074.

36. Shebuski RJ, Ramjit DR, Bencen GH, et al. Characterization and platelet inhibitory activity of bitistatin, a potent arginine-glycineaspartic acid-containing peptide from the venom of the viper Bitis arietans. J Biol Chem 1989; 264(36):21550-21556.

37. Hoole M, Goddard A. Boomslang envenomation in 2 dogs in KwaZulu-Natal, South Africa. J S Afr Vet Assoc 2007; 78(1):49-51.

38. Nilsson IM. Coagulation and fibrinolysis. Scand J Gastroenterol 1987; 22:11-18 